\title{
Network Pharmacology Integrated Molecular Docking Reveals The Mechanism of Huanglian Wendan Decotion Against Metabolic Syndrome
}

\section{Junli Bao}

Heilongjiang university of chinese medicine

\section{Xinyuan Gao}

Heilongjiang university of chinese medicine

\section{Yubo Han}

Heilongjiang university of chinese medicine

\section{Ke Zhang}

Heilongjiang University of Chinese Medicine

Li Liu ( $\nabla$ professorli.liu@hotmail.com )

Heilongjiang University of chinese medicine https://orcid.org/0000-0002-2490-2979

\section{Research}

Keywords: Huanglian Wendan Decotion, Metabolic Syndrome, Network Pharmacology, Molecular Docking

Posted Date: November 23rd, 2020

DOI: https://doi.org/10.21203/rs.3.rs-112113/v1

License: (c) (i) This work is licensed under a Creative Commons Attribution 4.0 International License. Read Full License 


\section{Abstract}

Background: Metabolic syndrome (MetS) is a group of abnormalities which includes abdominal obesity, hypertension, insulin resistance, and dyslipidemia. Many clinical studies showed that Huanglian Wendan Decoction(HWD) is effective in the treatment of MetS.

Methods: In this study, the Traditional Chinese Medicine Systems Pharmacology (TCMSP) database, STITCH database and Swisstargetpredicition database were used to excavate the active components of HWD and to predict the potential targets. The key targets of MetS are screened through GeneCards database and DisGeNET database. Moreover, we obtained the overlapping target of HWD and MetS based on venndiagram of "R program". Next, we performed enrichment analysis for common-target network and protein-protein interaction (PPI) network. Afterwards, Cytoscape was used to construct the components-targets core network for HWD in treating MetS, and R program was used to perform GO-BP and KEGG pathway enrichment analysis on these core targets. Last, we further investigated our predictions of crucial targets by performing molecular docking studies with components of HWD.

Results: The component-target core network of HWD against MetS was screened to contain 22 active components, which corresponded to 77 core targets. Additionally, enrichment analysis suggested that targets of HWD against MetS were mainly clustered into multiple biological processes (response to nutrient levels, gland development, response to steroid hormone, cellular response to oxidative stress, reproductive structure development etc and related pathways (AGE-RAGE, AMPK, JAK-STAT, MAPK), indicating the underlying mechanisms of HWD on MetS. The results of molecular docking showed that beta-sitost, naringenin, berberine and baicalein bound well to IL6 and AKT1.

Conclusion: The network pharmacological strategy integrated molecular docking to explore the mechanism of HWD against MetS. Our present outcomes might shed light on the further clinical application of HWD on MetS treatment.

\section{Introduction}

Metabolic syndrome (MetS) is a complex group of metabolic disorders which includes abdominal obesity, hypertension, insulin resistance, and dyslipidemia[1,2]. MetS is a risk factor leading to type 2 diabetes mellitus(T2DM) and cardiovascular disease(CVD) [3]. Patients who fulfil the criteria of MetS have a threefold increased risk of diabetes, and two-fold increased risk of CVD[4-5]. Epidemiological surveys show that MetS is a highly and increasingly prevalent medical condition[6], affecting well over $20 \%$ of the adult population in the USA[7], China[8], Europe[9], and the developing countries. The pathogenesis of MetS is still not completely clarified. Present studies have suggested that insulin resistance, adipose tissue dysfunction, chronic inflammation, oxidative stress, circadian disruption, microbiota, genetic factors might be involved in the pathogenesis of MetS[10]. The unknown pathogenesis has brought difficulties to the treatment, existing therapies to tackle various components of MetS are limited by various factors[11- 
13]. Traditional Chinese medicine (TCM) has unique advantages in treating Mets. In recent years, TCM has made good progress in the treatment of MetS[14-15].

Huanglian Wendan Decoction(HWD) is originated from "Liu Yin Bian Zheng", consisting of eight herbs: Coptis Chinensis(Huanglian HL), Aurantii Fructus Immaturus(Zhishi ZS), Poria Cocos(Schw.) Wolf(Fuling $\mathrm{FL}$ ), Citri Exocarpium Rubrum(Juhong JH), Zingiber Officinale Roscoe(Shengjiang SJ), Licorice(Gancao GC), Arum Ternatum Thumb(Banxian BX), Caulis Bambusae in Tania(Zhuru ZR)[16-17]. The effects of HWD on MetS have been validated by clinical practice in multiple years. However, the scientific basis as well as potential pharmacological mechanisms of HWD is still unclear, which has great limitations on the clinical effect of HWD[18].

Due to the large number of chemical components of chinese medicines, the interaction of multiple chinese medicines in the prescriptions, the long experimental period, and the heavy workload, it is impossible to comprehensively test the target of chinese medicines, nor to verify the all components through experiments. Herein, in this study, a comprehensive approach (network pharmacology combined with molecular docking) was utilized to probe the pharmacological mechanisms of HWD against MetS and providing ideas for subsequent research.

\section{Materials And Methods}

\subsection{Screening of Potential Pharmacological Active Components and Targets of HWD}

TCMSP[19](http://Isp.nwu.edu.cn/tcmsp.php) is a unique platform for systematic pharmacology of Chinese medicine, which can capture the relationship among drugs, targets and diseases. In order to obtain the information about the components of HWD, "Huang Lian, Zhi Shi, Fu Ling, Ju Hong, Sheng Jiang, Gan Cao, Ban Xia, and Zhu Ru"

were used as key words to search in theTCMSP database. Then, according to the principle of

pharmacokinetics absorption, distribution, metabolism and excretion in vivo, the drug screening threshold was set as $\mathrm{OB}$ (oral bioavailability) $\geq 30 \%$, DL (patent drug similarity) $\geq 0.18$. We also obtained potential targets protein names of compounds by TCMSP database. Then the candidate targets were mapped to Universal Protein[20] (Uniprot, http://www.uniprot.org/) for annotation and normalization. In addition, we also search Stitch database[21] (http://Stitch.embl.de/ver) and Swisstargetpredicition database[22] (http://www.swisstargetprediction.ch/) to supplement the targets of the compounds obtained by TCMSP database. To ensure the accuracy of the targets, set the status of the targets in the TCMSP database to be validated, the probility of the target in swisstargetprediction is 1 , and the combine score of the target in the Stitch database is greater than 0.99 .

\subsection{Collection of known MetS-related targets}

In order to obtain the known MetS-related targets, "metabolic syndrome" was used as key word to search in GeneCards database[23] and DisGeNET database[24]. After searching in the GeneCards database, 
results were sorted by the relevance score(RS), following by the removal of targets lower than the 20 . Additionally, MetS-related targets from DisGeNET database were sorted by the disease specificity index (DSI), following by the removal of targets lower than the median of DSI.

\subsection{Excavation of Core Targets of HWD for Treating MetS and Construction of Core Networks}

In order to obtain the candidate targets of HWD for treating MetS, we uploaded both potential targets of HWD and known MetS-related targets to the online Wayne diagram tool[25] (http://bioinfogp.cnb.csic.es/tools/venny/index.html) for overlapping targets. Subsequently, the candidate targets were used to construct a PPI network with multiple protein patterns on the Search Tool for the Retrieval of Interacting Genes/Proteins (STRING) platform[26] (https://string-db.org/). We set the organism type to "Homo sapiens" and left the default settings in place for the other parameters. Then, we exported the "string_interactions.tsv" file. Then, Cytoscape 3.7.2 software was used to construct two types of networks: components-targets network, protein-protein interaction network.

\subsection{Enrichment Analysis of Core Targets}

GO-biological process (BP) and KEGG pathway enrichment analysis are important methods used to describe the characteristics of candidate targets. We selected the standard p_value cutoff of 0.05 and the q_value of 0.05 and performed the enrichment analysis with $\mathrm{R}$ programe[27](Bioconductor, clusterProfiler).

\subsection{Molecular docking simulation}

Combining disease-related pathways, find out the differential genes in the pathway, and then query the corresponding compound molecules, download the 3D structure from the RCSB protein database[28] (http://www.pdb.org/). And use MOE software to modify it to remove ligands, add hydrogen, remove water, optimize and repair amino acids, and minimize the energy of all candidate targets. Finally, the above-mentioned core compound is used as a ligand, and the protein corresponding to the core target is used as a receptor for molecular docking.

\section{Results}

\subsection{Screening of Active Components and Their Targets of HWD}

Retrieved from TCMSP database, there were 173 related components of the whole formula in total by the screen criteria of OB and DL. The active components of HL, ZS, FL, JH, SJ, GC, BX, were 14, 22, 15, 9, 5, 92, 13 respectively. 3 active components of ZR were retrieved from Stich database(ZR is not included in the TCMSP database, only through literature or searching other databases to find the compound of $Z R$ ). 134 active components remained after removing no target genes and duplication. Subsequently, we explored the potential targets of the 134 potential pharmacologically active components by excavating TCMSP databases, Stitch database and Swisstargetpredicition database, which yielded to 219 targets after removing duplication(shown in_Table S1). 


\subsection{Excavation of the Core Targets of HWD in Treating MetS}

We collected 1728 targets(shown in Table S2) associated with MetS from the GeneCards database and DisGeNET database. The target genes of HWD were compared with the related genes of MetS and 77 overlapping common targets(shown in Table S3) were screened out. To explore the interaction between 77 overlapping common targets, we built a PPI network(Figure 1). The network had 76 nodes(One target had no interaction relationship with other targets, so the target was removed), which interacted with 817 edges, the average node degree is 21.5. As the degree increase, the nodes color deepens and size increase, the combine_score increase, the edges color deepens and thicker increase, which suggest stronger interactions. These results demonstrate that these targets were defined as the candidate targets for HWD in treating MetS.

\subsection{Construction of Components-Targets Core Network for HWD in Treating MetS}

In order to holistically and systemically obtain comprehensive understanding of the component -target for HWD in treating MetS, a network map was constructed by using Cytoscape. First, we found the corresponding components of 77 core targets. The basic information of the components of HWD is shown in Table 1. Then we used Cytoscape to construct a network map, including 301 edges and 115 nodes (igure 2). It showed that HWD plays a therapeutic role in MetS mainly through multi-component corresponding to multi-target.

Table 1. active components of HWD. 


\begin{tabular}{|c|c|c|c|c|c|}
\hline MOL ID & Component name & OB\% & DL & Number of targets & Herb \\
\hline MOL000098 & quercetin & 46.43 & 0.28 & 84 & $\mathrm{GCHL}$ \\
\hline MOL000289 & pachymic acid & 33.63 & 0.81 & 1 & $\mathrm{FL}$ \\
\hline MOL000354 & isorhamnetin & 49.6 & 0.31 & 5 & $\mathrm{GC}$ \\
\hline MOL000358 & beta-sitosterol & 36.91 & 0.75 & 3 & $\mathrm{BX} \mathrm{JH}$ \\
\hline MOL000392 & formononetin & 69.67 & 0.21 & 5 & $\mathrm{GC}$ \\
\hline MOL000417 & Calycosin & 47.75 & 0.24 & 1 & $\mathrm{GC}$ \\
\hline MOL000422 & kaempferol & 41.88 & 0.24 & 8 & $\mathrm{GC}$ \\
\hline MOL000449 & stigmasterol & 43.83 & 0.76 & 4 & BX SJ \\
\hline MOL000497 & licochalcone a & 40.79 & 0.29 & 2 & $\mathrm{GC}$ \\
\hline MOL001454 & berberine & 36.86 & 0.78 & 1 & $\mathrm{HL}$ \\
\hline MOL001803 & sinensetin & 50.56 & 0.45 & 1 & ZS \\
\hline MOL001942 & isoimperatorin & 45.46 & 0.23 & 4 & JH \\
\hline MOL002670 & Cavidine & 35.64 & 0.81 & 1 & $B X$ \\
\hline MOL002714 & baicalein & 33.52 & 0.21 & 10 & $B X$ \\
\hline MOL002776 & baicalin & 40.12 & 0.75 & 1 & $B X$ \\
\hline MOL004328 & naringenin & 22.05 & 0.74 & 24 & $\mathrm{GC}$ \\
\hline MOL004804 & 18beta-glycyrrhetinic acid & 59.29 & 0.21 & 1 & GC CP ZS JH \\
\hline MOL005812 & naringin & 22.05 & 0.74 & 1 & $\mathrm{GC}$ \\
\hline \multirow[t]{4}{*}{ MOL005828 } & nobiletin & 61.67 & 0.52 & 1 & ZS CP \\
\hline & arginine & & & 3 & ZR \\
\hline & glutamic acid & & & 3 & ZR \\
\hline & ornithine & & & 3 & ZR \\
\hline
\end{tabular}

\subsection{Enrichment Analysis of the Core Targets of HWD in Treating MetS}

In order to further understand the mechanism of "multitarget and multipathway" of HWD in treating MetS, "R program" was used to perform enrichment analysis of GO-PB and KEGG on core targets and to excavate the biological processes and signaling pathways regulated by HWD in treatingMetS. These 77 core targets were involved in several biological process, mainly including response to nutrient levels, gland development, response to steroid hormone, cellular response to oxidative stress, reproductive 
structure development et al(Figure 4). Moreover, according to the p_values of enriched pathways and their correlation with MetS(shown in Table S4), we were most interested in the following five representative signal pathways including AGE-RAGE, MAPK, AMPK, JAK-STAT signaling pathways (Table 2).

Table 2 Representative enriched KEGG pathway of the core targets of HWD in treating Mets.

\begin{tabular}{|lllll|}
\hline Pathway & Gene count & P value & Pathway ID & Associated genes \\
\hline AGE-RAGE signaling pathway & 9 & $1.93 \mathrm{E}-07$ & hsa04933 & \\
\hline AMPK signaling pathway & 8 & $9.39 \mathrm{E}-06$ & hsa04152 & \\
\hline JAK-STAT signaling pathway & 8 & $8.22 \mathrm{E}-05$ & hsa04630 \\
\hline MAPK signaling pathway & 12 & $4.91 \mathrm{E}-08$ & hsa04010 \\
\hline
\end{tabular}

\subsection{Predicted binding of components of HWD to target proteins in MetS}

To further validate candidate compounds of HWD targets in MetS, we tested the precision of docking between beta-sitost, naringenin, berberine, baicalein and the following potential target proteins (Figure45): IL6(PDB:6NCO) and AKT1(PDB:5KCV). We chose to study these target proteins because they were high-degree nodes in the network with many functional connections. Meanwhile, there were enriched in related-pathways, suggesting they play a critical role in the response to compounds in MetS. Docking analysis successfully predicted docking between beta-sitost, naringenin, berberine, baicalein and the binding pocket of two tested target proteins. Overall, these results provide further evidence that these two proteins act as beta-sitost, naringenin, berberine and baicalein targets in MetS.

\section{Discussion}

HWD is an common prescription for MetS at the Department of Endocrinology in our hospital. It is especially suitable for patients with phlegm-heat subtype of MetS, with obesity, bitter mouth, dry mouth, red tongue, and yellow fur[29]. The therapeutic effects of HWD in the clinical treatment of MetS are significant[30]. However, the active compounds and potential targets of HWD are unclear, which hinders the further development and application of the prescription. In this study, network pharmacology combined with molecular docking approach were applied to determine the underlying mechanisms of HWD in MetS.

In this study, a total of 22potential active components of HWD in treating MetS were screened through a series of network pharmacological methods. Beta-sitosterol, naringenin, quercetin, stigmasterol and nobiletin are common compounds of two or more Chinese medicines. Studies have shown that betasitosterol alone can increase plasma adiponectin concentration and reduced plasma insulin 
concentration and homeostatic model assessment index[31]. Naringenin supplementation has proven to be efficacious for the treatment of metabolic syndrome and obesity in animal models[32]. Naringenin may be useful for ameliorating the inflammatory changes in obese adipose tissue. Recent researches have demonstrated berberine can protects rats from MetS through exerting inhibition on the HPA-axis and increasing skeletal muscle expression of GLUT4 proteins[33]. Research showed baicalein protects mice from MetS through an AMPKa(2)-dependent mechanism involving multiple intracellular signaling pathways[34]. The compound-target network diagram (Fig. 2) shows that there was a complex network relationship between the compounds and the targets.

In the PPI network, 77 core targets are important target proteins for the HWD as used to treat MetS. The top 5 targets(INS, IL6, AKT1, VEGFA, ESR1) ranked by degree have been confirmed to be closely related to the occurrence and development of MetS. INS gene mutations have been confirmed to be closely related to the occurrence of MetS[35]. Studies have shown that IL-6 is involved in the occurrence and development of all components in MetS such as hypertension, insulin resistance, obesity and lipid dysmetabolism[36]. AKT1 is a key mediator in the insulin-signaling pathway which has been proven to be important target proteins for the HWD as used to treat MetS[37]. Recent studies have suggested that the VEGFA gene plays an important role in the pathogenesis of MetS and its related disorders. Targeting VEGFA and its inhibition and blockage have been suggested as novel therapeutic approaches to improve MetS.

Enrichment analysis of GO-BP and KEGG on the core targets further suggests that HWD could intervene in MetS through multiple biological processes by acting on several signaling pathways, involving AGERAGE, AMPK, JAK-STAT, MAPK signaling pathways. Luca Cannizzaro used samples taken from different body fluids and tissues of a rat model of high-fructose diet-induced MetS. The results showed that the following molecular parameters of the AGE-RAGE signaling pathway were significantly upregulated. Recent researches have demonstrated AMPK activation to ameliorate multiple components of metabolic syndrome by regulating a balance between anabolic and catabolic cellular reactions. Recent researches have demonstrated that dysregulation of the JAK-STAT pathway would lead to a multiple metabolism disorders and medicines for this signaling pathway maybe become a new idea for MetS. Animal experiment have shown spironolactone treatment administered inhibited phosphorylation of p38MAPK, which inhibit pancreatic gland $\beta$-cell apoptosis in MetS. This indicates P38MAPK pathways is the treatment target of MetS.

\section{Conclusions}

Our team has previously confirmed the safety and effectiveness of HWD in treating MetS through clinical observations. Network pharmacology and molecule docking method confrms the "multicompounds, multitargets" therapeutic actions of HWD in the treatment of MetS. The present work may provide valuable evidence for further clinical application of HWD for treating MetS and may lay a good theoretical foundation for further experimental verification and facilitate the widespread application of HWD in treating MetS. 


\section{Abbreviations}

metabolic syndrome: MetS

cardiovascular disease: CVD

type 2 diabetes mellitus: T2DM

protein-protein interaction: PPI

Huanglian Wendan decoction: HWD

Traditional Chinese Medicine Systems Pharmacology Database: TCMSP

oral bioavailability: OB

druglikeness: DL

Search Tool for Interactions of Chemicals: STITCH

Universal Protein: Uniprot

Search Tool for the Retrieval of Interacting Genes/Proteins: STRING

biological process: $\mathrm{BP}$

\section{Declarations}

\section{Data Availability}

The data used to support the findings of this study are available from the corresponding author upon request.

\section{Disclosure}

The funders had no role in study design, data collection and analysis, decision to publish, or preparation of the manuscript.

\section{Conflicts of Interest}

The authors declare that they have no conflicts of interest.

\section{Authors' Contributions}

Junli Bao designed the study, analyzed the data, and wrote the manuscript; Li Liu supervised the study and revised the manuscript; Yubo Han, Xinyuan Gao and Ke Zhang obtained and analyzed the data. All 
authors have read and agreed to publish this manuscript.

\section{Acknowledgments}

This is work was supported by National Natural Science Foundation of China (81873284).

\section{References}

1. Vanlancker T, Schaubroeck E, Vyncke K, et al. Comparison of definitions for the metabolic syndrome in adolescents: the HELENA study. Eur J Pediatr. 2017;176(2):241-252.

2. Rochlani Y, Pothineni NV, Kovelamudi S, et al. Metabolic syndrome: pathophysiology, management, and modulation by natural compounds. Ther Adv Cardiovasc Dis. 2017;11(8):215-225.

3. Pucci G, Alcidi R, Tap L, et al. Sex- and gender-related prevalence, cardiovascular risk and therapeutic approach in metabolic syndrome: A review of the literature. Pharmacol Res. 2017;120:34-42.

4. Ford ES. Risk for All-Cause Mortality, Cardiovascular Disease, and Diabetes Associated with the Metabolic Syndrome. A summary of the evidence. Diabetes Care. 2005;28(7):1769-1778.

5. Mottillo S, Filiion KB, Genest J, et al. The Metabolic Syndrome and Cardiovascular Risk. A systematic review and meta-analysis. J Am Coll Cardiol. 2010;56(14):1113-1132.

6. Haffner S, Taegtmeyer H. Epidemic obesity and the metabolic syndrome. Circulation. 2003;108(13): 1541-1545.

7. Beltrán-Sánchez H, Harhay MO, Harhay MM, et al. Prevalence and trends of metabolic syndrome in the adult U.S. population, 1999-2010. J Am Coll Cardiol. 2013;62(8):697-703.

8. Xi B, He D, Hu Y, Zhou D. Prevalence of metabolic syndrome and its influencing factors among the Chinese adults: the China Health and Nutrition Survey in 2009. Prev Med. 2013;57(6):867-871.

9. Vishram JKK, Borglykke A, Andreasen AH, et al. Impact of age and gender on theprevalence and prognostic importance of the metabolic syndrome and its components in Europeans. The MORGAM Prospective Cohort Project. PLoS One. 2014;9(9):e107294.

10. Xu H, Li XH, Adams H, et al. Etiology of Metabolic Syndrome and Dietary Intervention. International Journal of Molecular Sciences. Int J Mol Sci. 2018;20(1):128.

11. Rochlani Y, Pothineni NV, Kovelamudi S, et al. Metabolic syndrome: pathophysiology, management, and modulation by natural compounds. Ther Adv Cardiovasc Dis. 2017 Aug;11(8):215-225.

12. Jahandideh F, Wu J. Perspectives on the Potential Benefits of Antihypertensive Peptides towards Metabolic Syndrome. Int J Mol Sci. 2020;21(6):2192.

13. Lim S, Eckel RH. Pharmacological treatment and therapeutic perspectives of metabolic syndrome. Rev Endocr Metab Disord. 2014;15(4):329-41.

14. Jiang YP, Liu JC, Li ZH, et al. Huanglian Wendan decoction on phlegm heat metabolic syndrome clinical curative effect observation. J Tianjin Univ Tradit Chin Med. 2018; 37(37): 287-291 (In Chinese). 
15. Liu HS, Liu L, Sui YB. Clinical study of Modified Huanglian Wendan Decoction on metabolic syndrome of phlegm and dampness. J Emerg in Tradit Chin Med. 2016;25(10):1839 (In Chinese).

16. Zhang FL, Fan LL, Li H,et al. On the six factors Clinical application of Huanglian Wendan Decoction. J Hunan Tradit Chin Med. 2018;vol.34(11):106-107 (In Chinese).

17. Du S, Zhao KW, Liu ZH, et al. Research progress and related thinking of Wendan Decoction. J Basic Tradit Chin Med. 2019;25(12): 1764-1768 (In Chinese).

18. Li YT, Fan LL, Yuan Y, et al. Research progress in pharmacological action and clinical application of Huanglian Wendan Decoction. Acta Chin Med Pharm. 2018;46(2): 115-119 (In Chinese).

19. Ru J, Li P, Wang J, et al. TCMSP: a database of systems pharmacology for drug discovery from herbal medicines. J Cheminform. 2014;6(1):13.

20. Chandran U, Patwardhan and B. Network ethnopharmacological evaluation of the immunomodulatory activity of Withania somnifera. J Ethnopharmacol. 2017;197: 250-256.

21. Kuhn M, Szklarczyk D, Pletscher-Frankild S, et al. STITCH 4: integration of protein-chemical interactions with user data. Nucleic Acids Res. 2014;42(1):D401-D407.

22. Antoine $D$, Olivier $M$, Vincent $Z$. SwissTargetPrediction: updated data and new features for efficient prediction of protein targets of small molecules. Nucleic Acids Res. 2019; W1:W1.

23. Stelzer G, Rosen N, Plaschkes I, et al. The GeneCards Suite: From Gene Data Mining to Disease Genome Sequence Analyses. Curr Protoc Bioinformatics. 2016; 54: 1-30.

24. Janet $P$, Núria OR, Àlex $B$, et al. DisGeNET: a discovery platform for the dynamical exploration of human diseases and their genes. Database (Oxford). 2015:bav028.

25. Lam F, Lalansingh CM, Babaran HE, et al. VennDiagramWeb: a web application for the generation of highly customizable Venn and Euler diagrams. BMC Bioinformatics; 2016;17(1):401.

26. Yang K, Gao J, Luo M. Identification of key pathways and hub genes in basal-like breast cancer using bioinformatics analysis. Onco Targets Ther. 2019;12:1319-1331.

27. Lu WD, Li L, Shen Y, et al. Network pharmacology study of Xiaoxuming Decoction based on vasodilatory and vasoconstrictory related GPCR targets. China Journal of Chinese Materia Medica. 2018; 43(23): 4698-4708.

28. Burley SK, Berman HM, Christie C, et al. RCSB Protein Data Bank: Sustaining a living digital data resource that enables breakthroughs in scientific research and biomedical education. Protein Sci. 2018; 27(1):316-330.

29. Wang Q, Liu L. Research progress of traditional Chinese medicine in the treatment of metabolic syndrome. J Inf Tradit Chin Med. 2017;6:142-145

30. Liu L, Li WZ, Zou GL, et al. Research progress of Huanglian Wendan Decoction in the treatment of metabolic syndrome. Chin Exp Tradit Med Form. 2019;17: 190-196.

31. Gumede NM, Lembede BW, Brooksbank RL, et al. $\beta$-Sitosterol Shows Potential to Protect Against the Development of High-Fructose Diet-Induced Metabolic Dysfunction in Female Rats. J Med Food. 2020;23(4): 367-374. 
32. Alam MA, Subhan N, Rahman MM. Effect of Citrus Flavonoids, Naringin and Naringenin, on Metabolic Syndrome and Their Mechanisms of Action. Adv Nutr. 2014;5(4):404-417.

33. Mi J, He W, Lv J, et al. Effect of berberine on the HPA-axis pathway and skeletal muscle GLUT4 in type 2 diabetes mellitus rats. Diabetes Metab Syndr Obes. 2019;12: 1717-1725,.

34. Pu P, Wang XA, Salim M, et al. Baicalein, a natural product, selectively activating AMPKa 2 and ameliorates metabolic disorder in diet-induced mice. Mol Cell Endocrinol. 2012;362(1-2):128-138.

35. Nicola S , Grazia C , Alessandra A, et al. Insulin Gene Variable Number of Tandem Repeats (INS VNTR) Genotype and Metabolic Syndrome in Childhood Obesity. J Clin Endocrinol Metab. 2006;91(11):4641-4644.

36. Testa R, Olivieri F, Bonfigli A, et al. Interleukin-6-174 G>C polymorphism affects the association between IL-6 plasma levels and insulin resistance in type 2 diabetic patients. Diabetes Res Clin Pract. 2006;71(3):299-305.

37. Bao PL, Liu GL, Wei Y. Association between IL-6 and related risk factors of metabolic syndrome and cardiovascular disease in young rats. International Journal of Clinical and Experimental Medicine. Int J Clin Exp Med. 2015; 8(8):13491-13499.

38. Kim Y, Cho Y, Kim D, et al. Metformin ameliorates IL-6-induced hepatic insulin resistance via induction of orphan nuclear receptor small heterodimer partner (SHP) in mouse models. Diabetologia. 2012;55(5):1482-1494.

39. Harmon BT, Devaney SA, Gordish-Dressman H, et al. Functional characterization of a haplotype in the AKT1 gene associated with glucose homeostasis and metabolic syndrome. Hum Genet. 2010;128(6):635-45.

40. Krook A, Roth RA, Jiang XJ. Insulin-stimulated Akt kinase activity is reduced in skeletal muscle from NIDDM subjects. Diabetes. 1998; 47(8):1281-1286,

41. Barylski $M$, Kowalczyk E, Banach $M$, et al. Plasma total antioxidant activity in comparison with plasma NO and VEGF levels in patients with metabolic syndrome. Angiology. 2009;60(1):87-92.

42. Siervo M, Ruggiero D, Sorice R, et al. Body mass index is directly associated with biomarkers of angiogenesis and inflammation in children and adolescents," Nutrition. 2012;28(3):262-266.

43. Wu LE, Meoli CC, Mangiafico SP, et al. Systemic vascular endothelial growth factor-A (VEGFA)neutralization ameliorates diet induced metabolic dysfunction. Diabetes. 2014;63(8):26562667,

44. Cannizzaro L, Rossoni G, Savi F, et al. Regulatory landscape of AGE-RAGE-oxidative stress axis and its modulation by PPARy activation in high fructose diet-induced metabolic syndrome. Nutr Metab (Lond). 2017;14:5.

45. Sharma H, Kumar S. Natural AMPK Activators: An Alternative Approach for the Treatment and Management of Metabolic Syndrome. Curr Med Chem. 2017;24(10):1007-1047.

46. Xu D, Yin C, Wang S, et al. JAK-STAT in lipid metabolism of adipocytes. JAK-STAT. 2013; 2(4):e27203. 
47. Long HD, Lin YE, Liu MJ, et al. Spironolactone prevents dietary-induced metabolic syndrome by inhibiting PI3-K/Akt and p38MAPK signaling pathways. J Endocrinol Invest. 2013;36(11):923-930,.

\section{Figures}

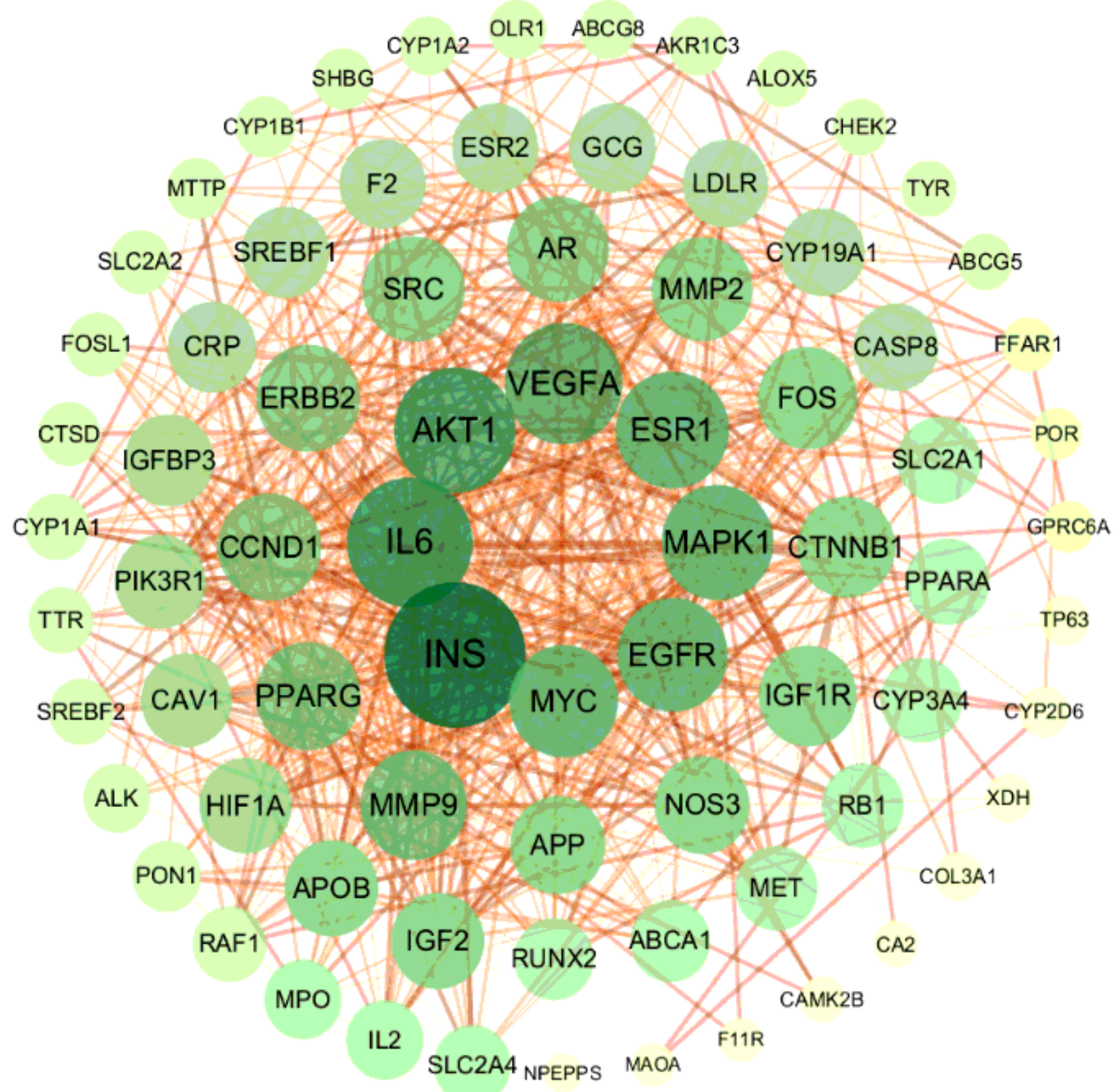

Figure 1

The PPI network of all the candidate targets of HWD in treating MetS 


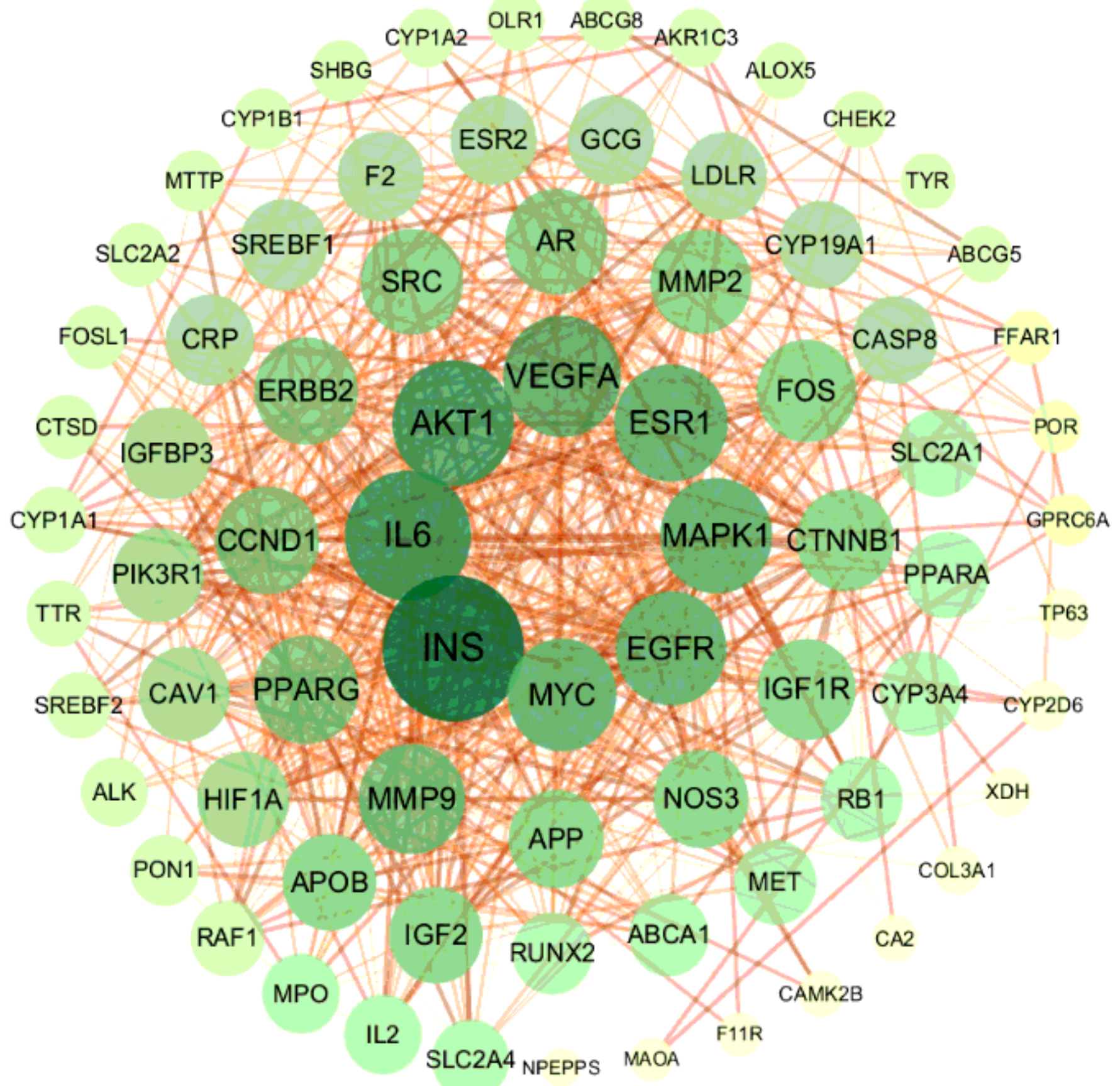

Figure 1

The PPI network of all the candidate targets of HWD in treating MetS 


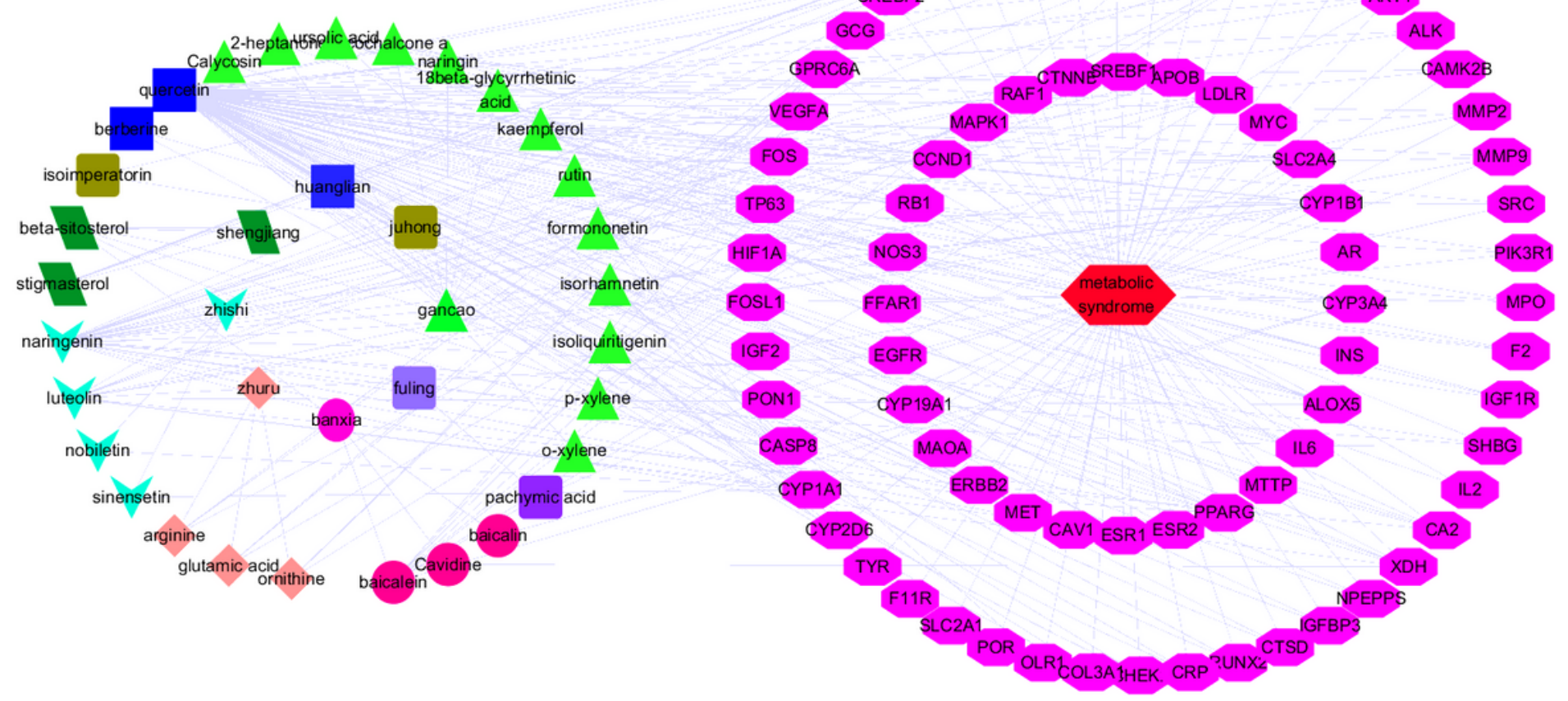

Figure 2

Construction of the HWD component-target network. The component-potential target network was constructed by linking the candidate compounds and their potential targets of the 8 herbs, which are constituents of HWD. The nodes on the left outer circle representing candidate components, the nodes on the left inner circle representing herbs and the targets are indicated by purple circle on the right.

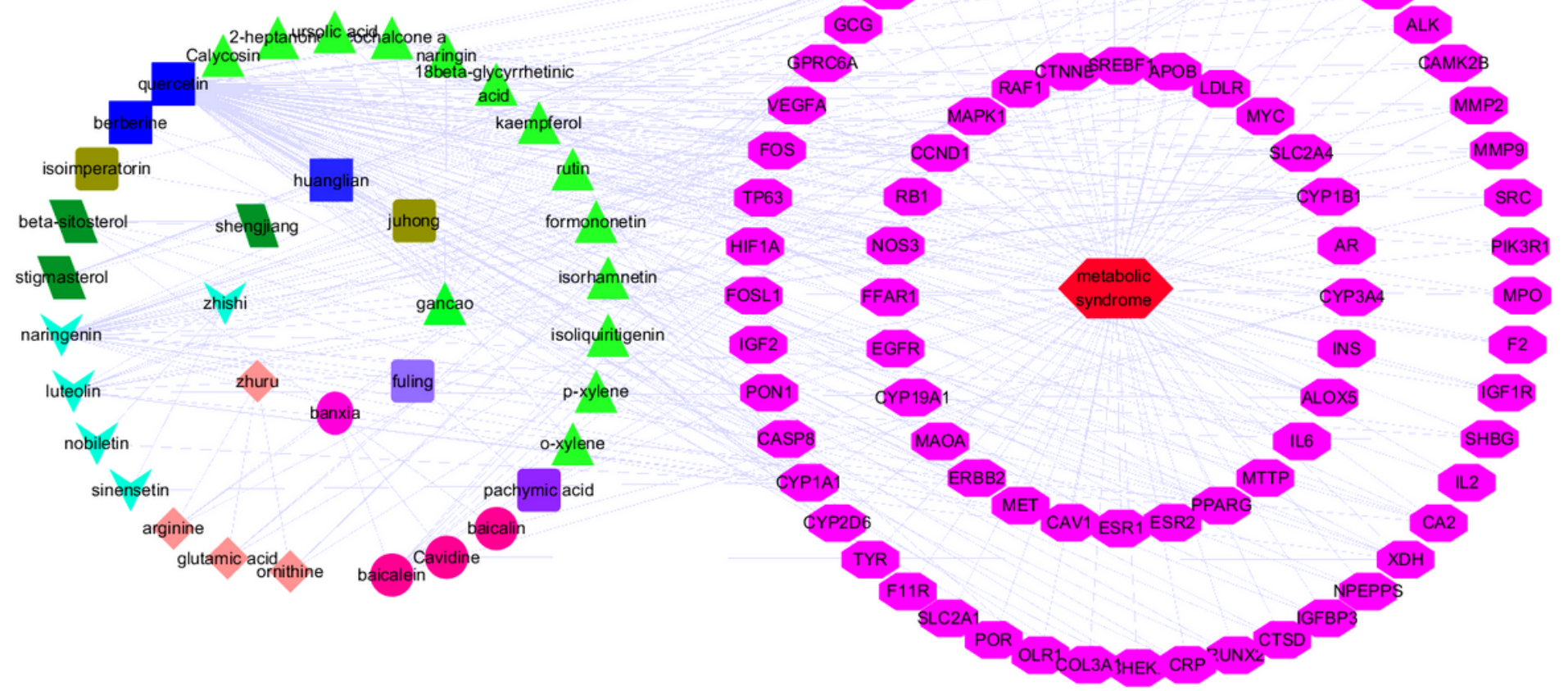


Figure 2

Construction of the HWD component-target network. The component-potential target network was constructed by linking the candidate compounds and their potential targets of the 8 herbs, which are constituents of HWD. The nodes on the left outer circle representing candidate components, the nodes on the left inner circle representing herbs and the targets are indicated by purple circle on the right.

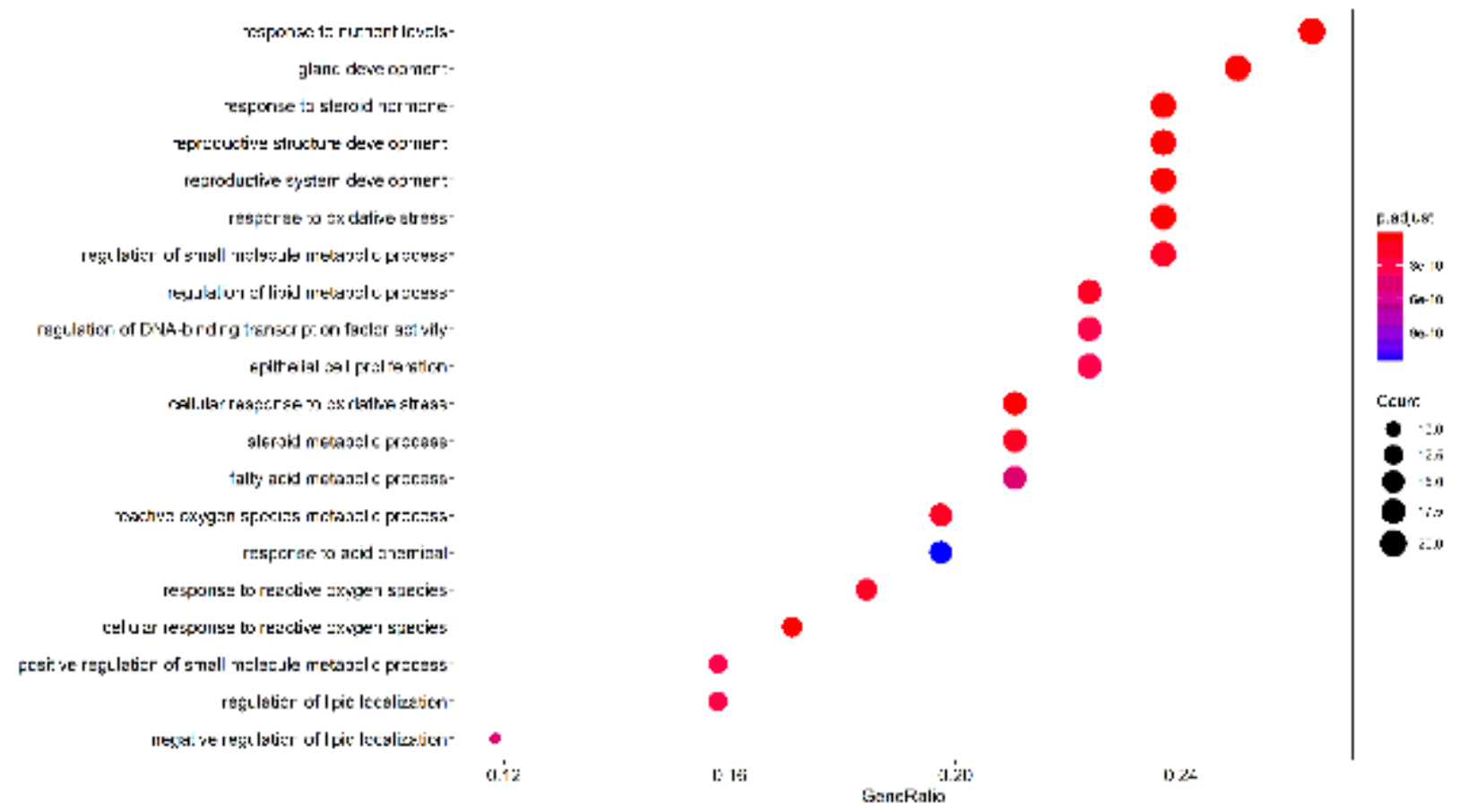

Figure 3

Bubble chart of GO functional enrichment for candidate targets.

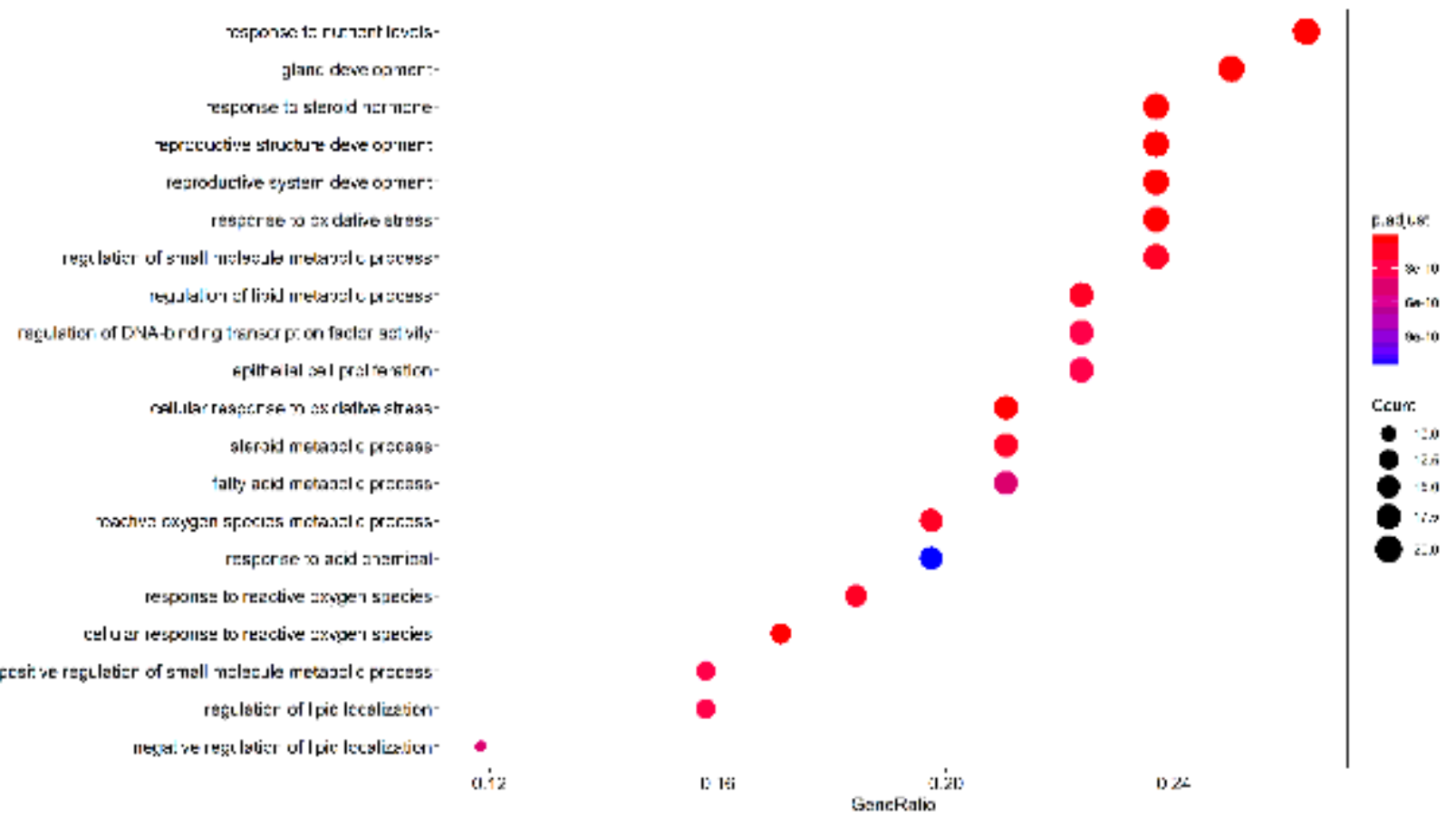


Figure 3

Bubble chart of GO functional enrichment for candidate targets.

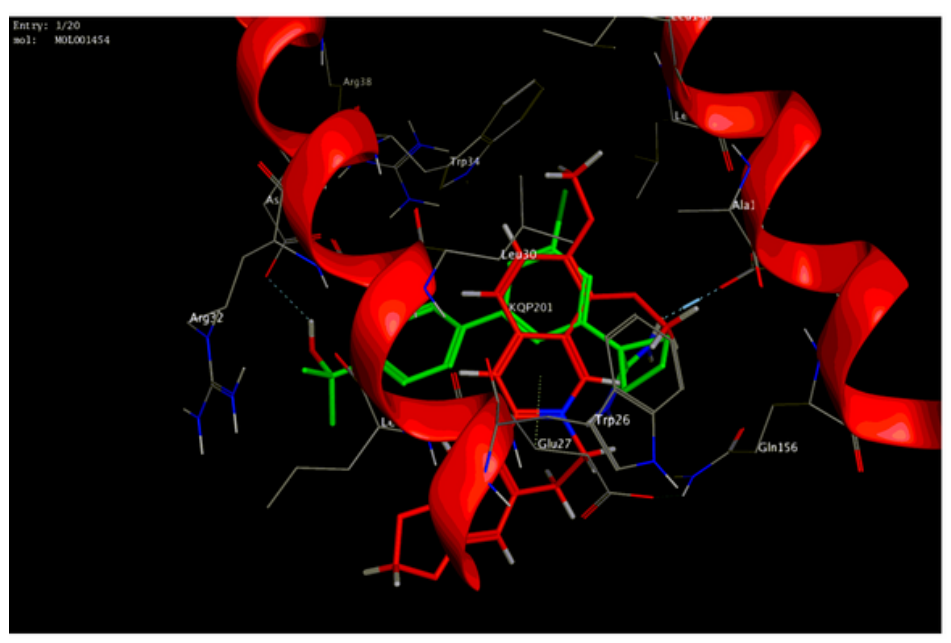

(a)

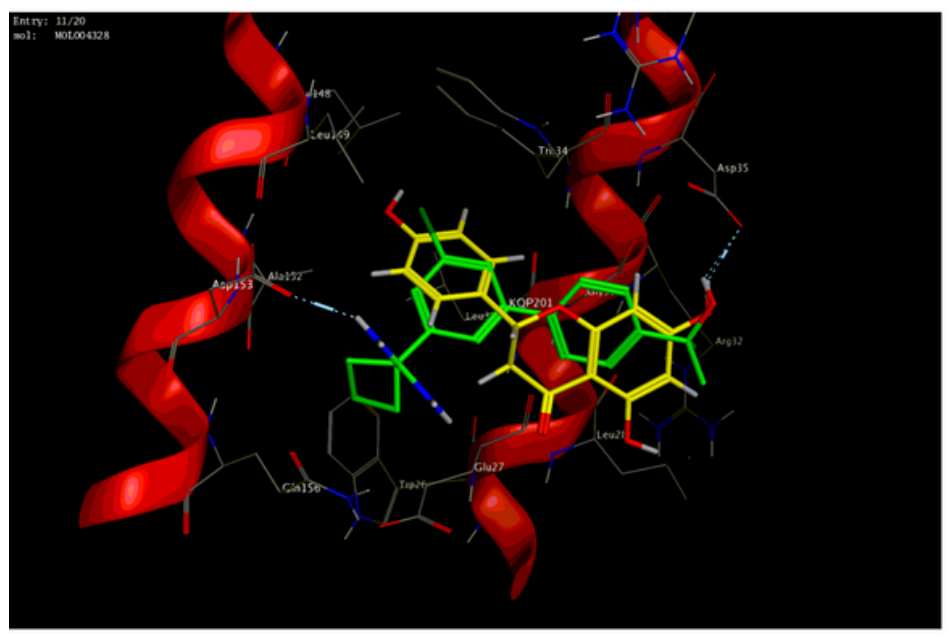

(c)

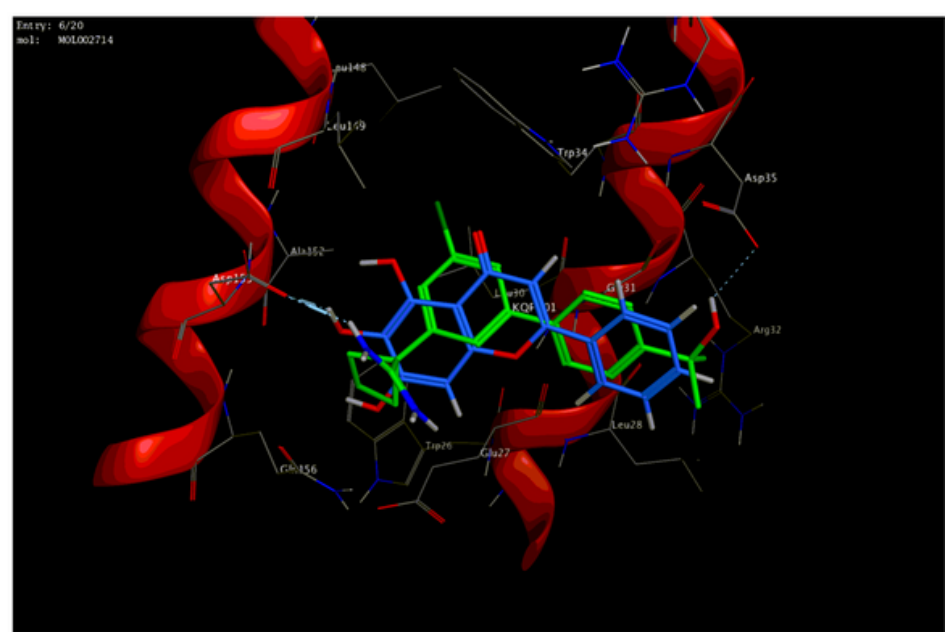

(b)

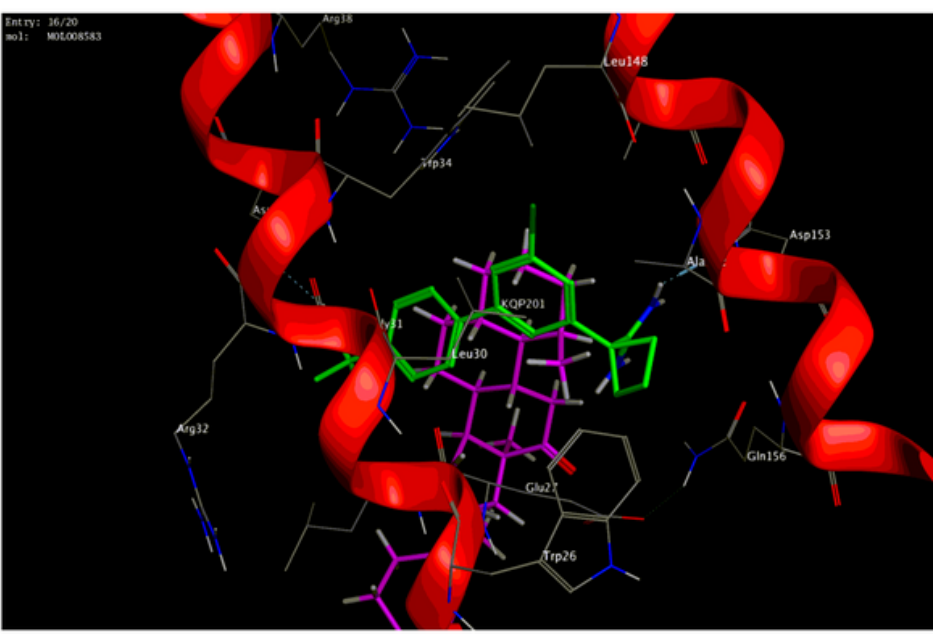

(d)

\section{Figure 4}

Molecular models of, naringenin, beta-sitosterol, baicalein, and berberine binding to the predicted target proteins AKT1. (a) AKT1-berberine, (b) AKT1- baicalein, (c) AKT1- naringenin, (d) AKT1- beta-sitosterol 


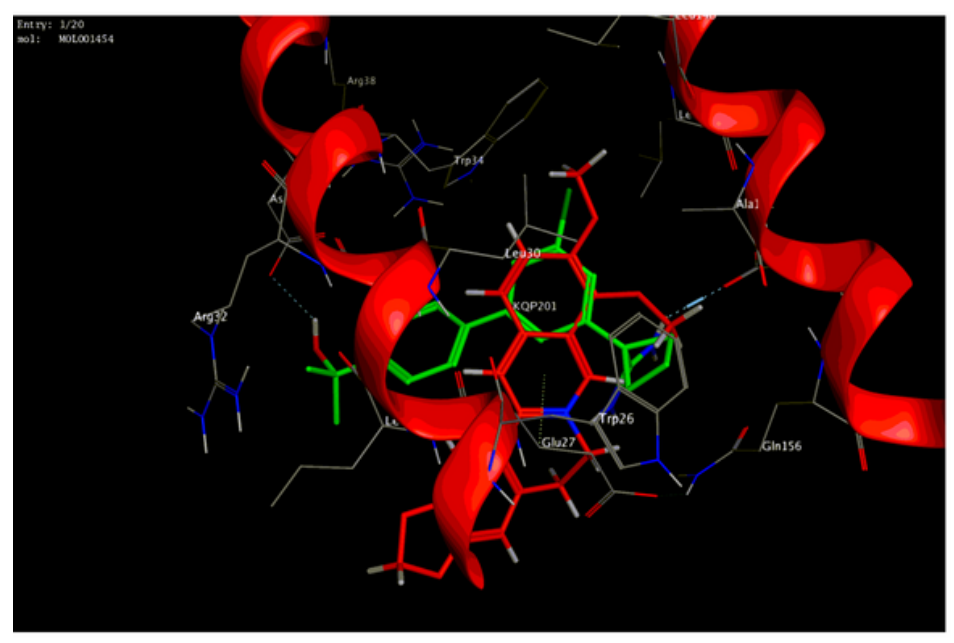

(a)

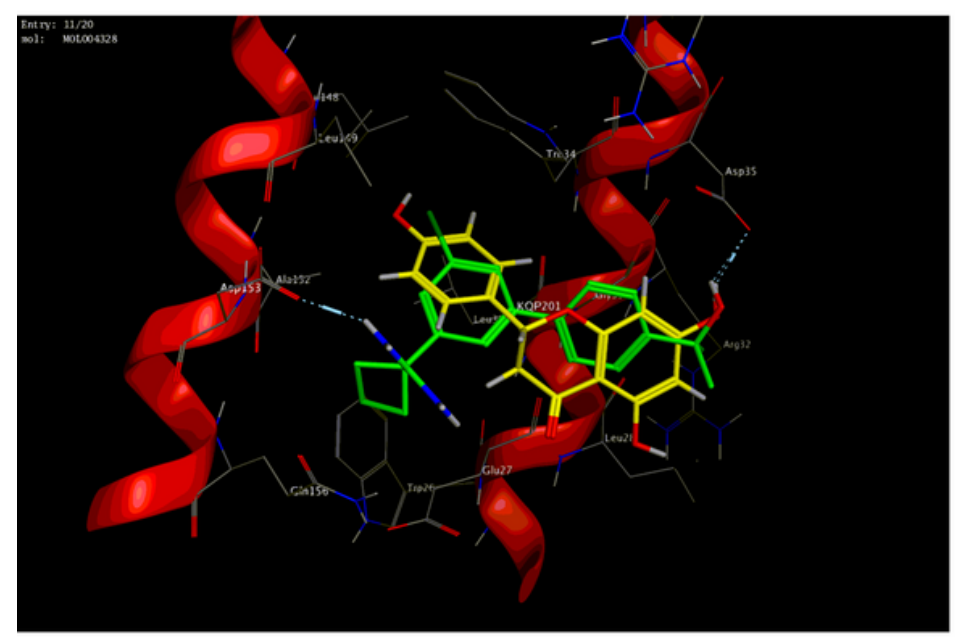

(c)

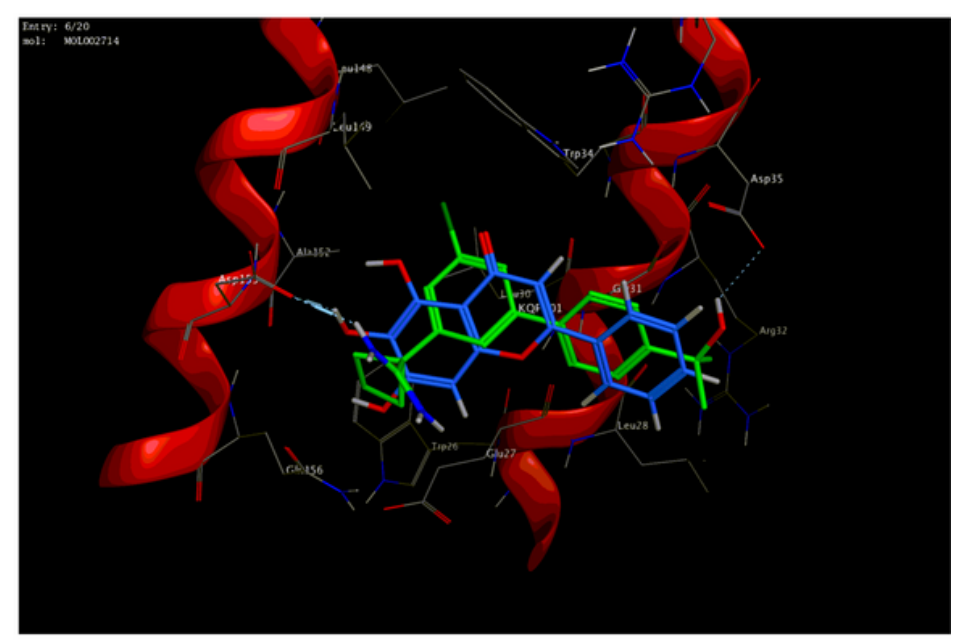

(b)

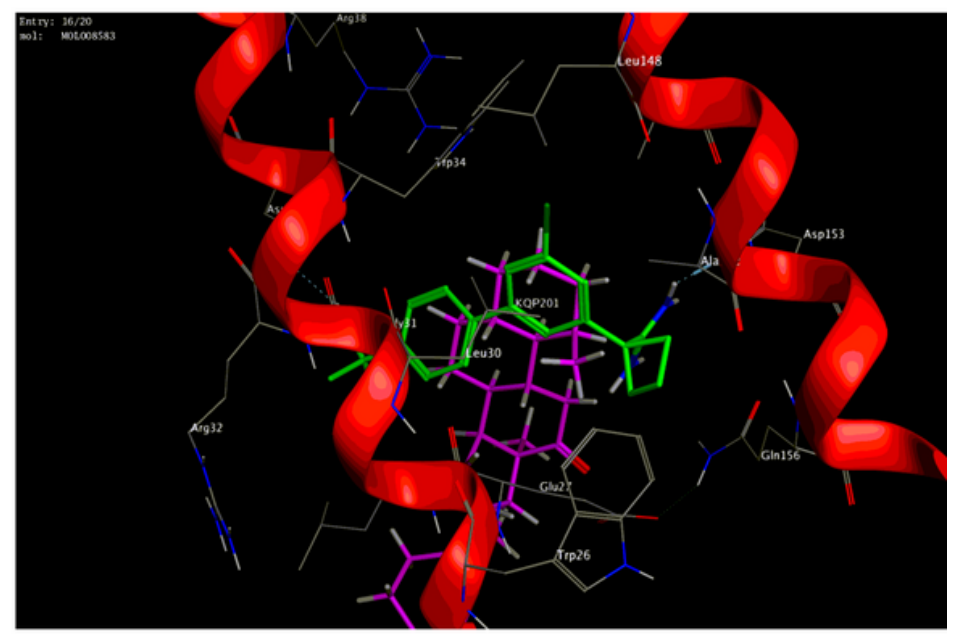

(d)

\section{Figure 4}

Molecular models of, naringenin, beta-sitosterol, baicalein, and berberine binding to the predicted target proteins AKT1. (a) AKT1-berberine, (b) AKT1- baicalein, (c) AKT1- naringenin, (d) AKT1- beta-sitosterol 


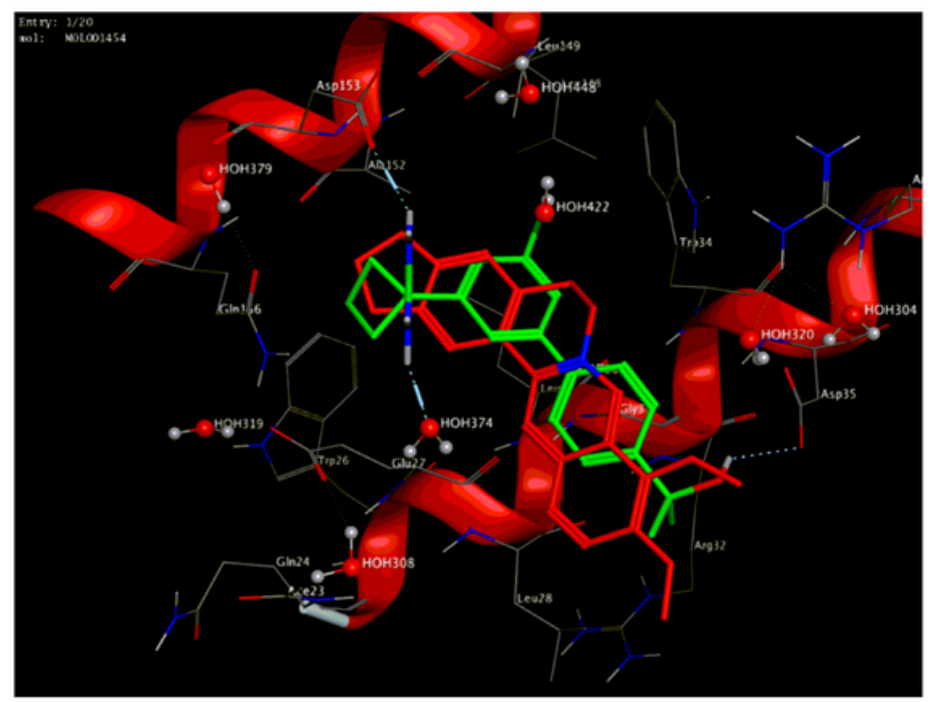

(a)

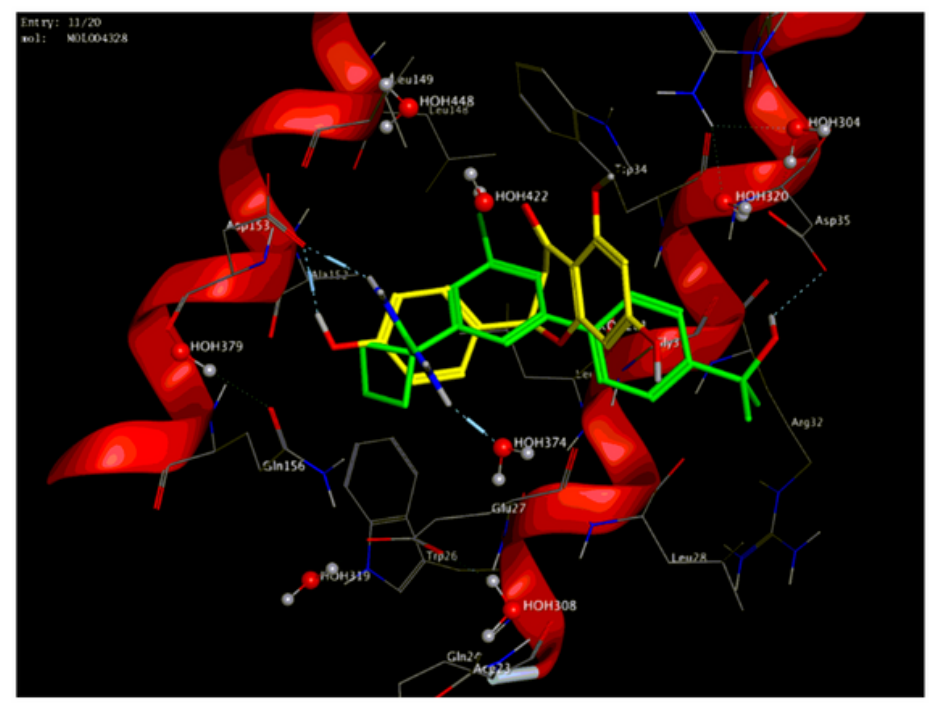

(c)

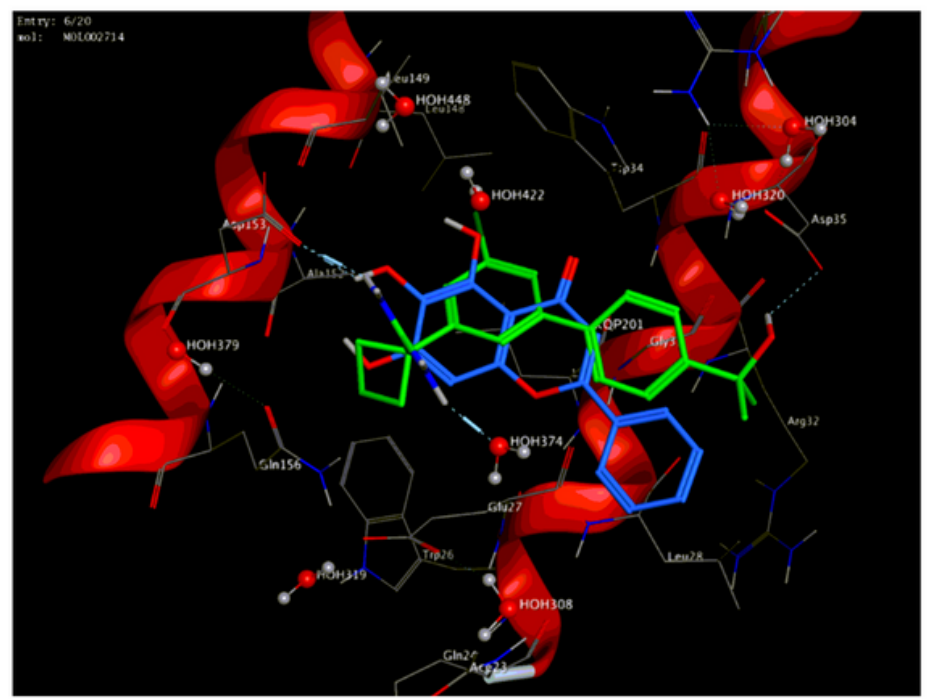

(b)

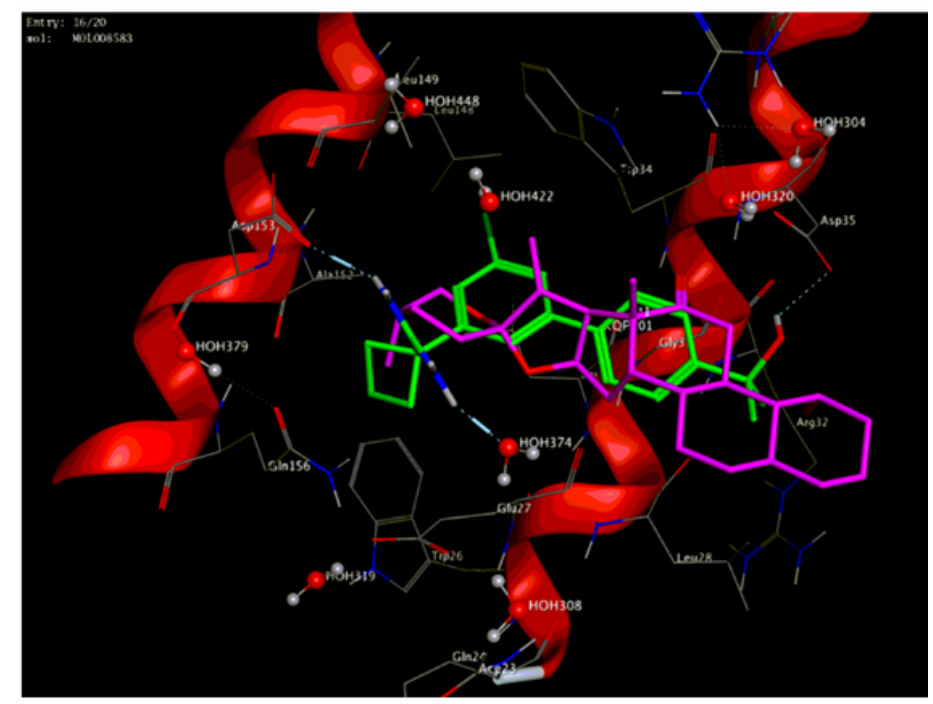

(d)

\section{Figure 5}

Molecular models of beta-sitosterol, naringenin, baicalein, and berberine binding to the predicted target proteins IL-6. (a) IL-6-berberine, (b) IL-6- baicalein, (c) IL-6- naringenin, (d) IL-6- beta-sitosterol 


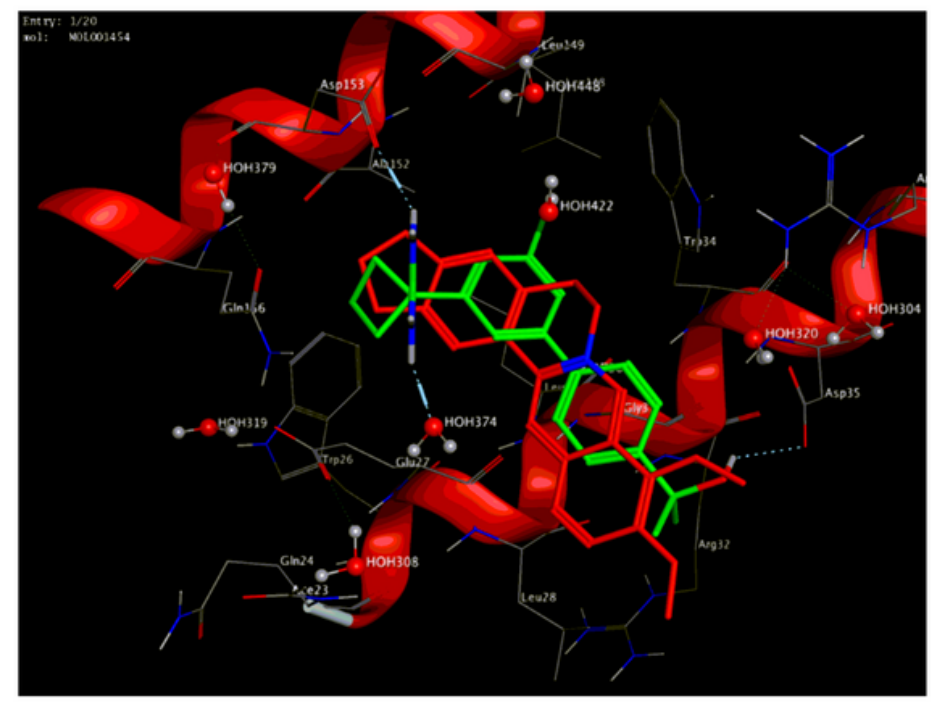

(a)

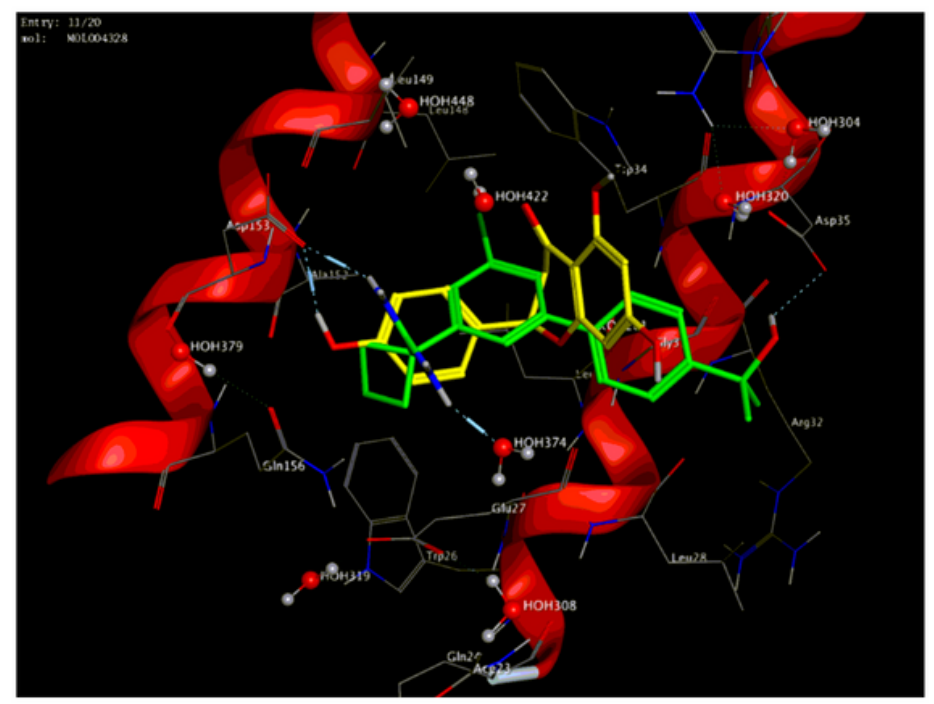

(c)

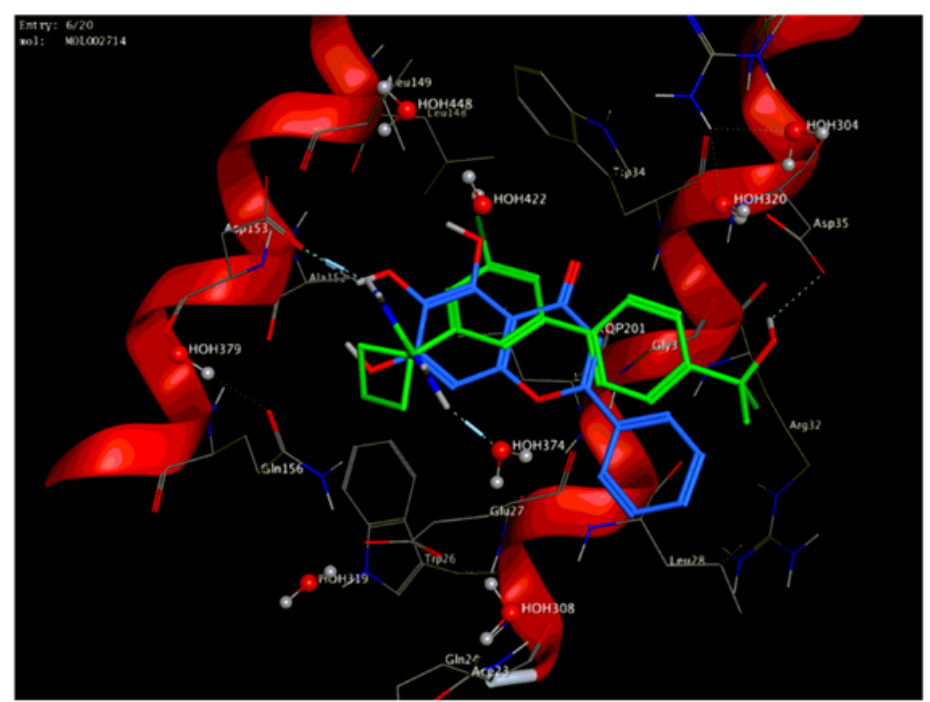

(b)

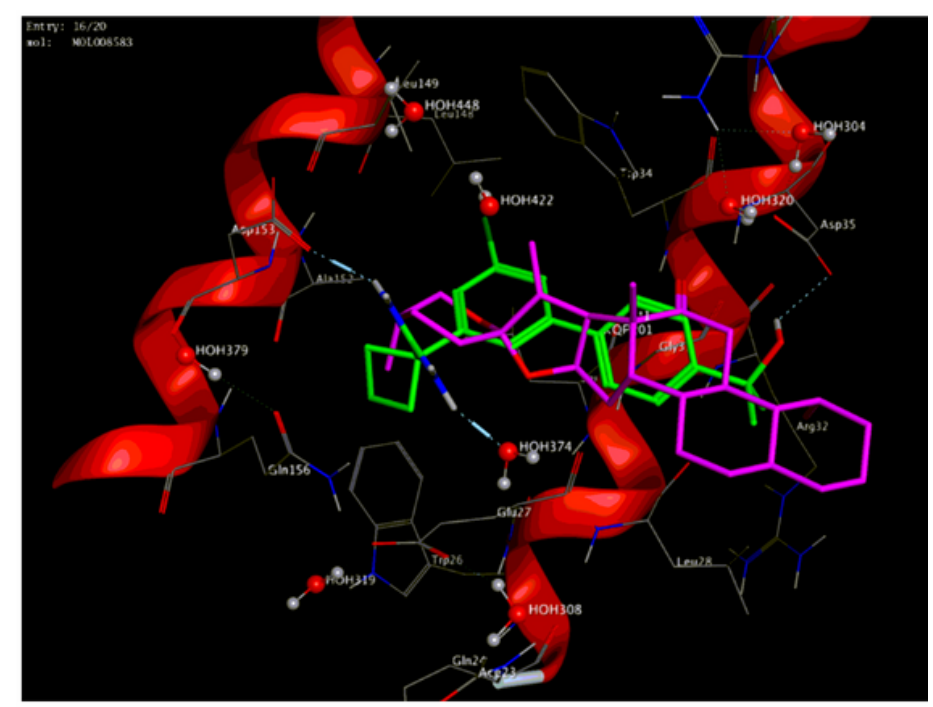

(d)

\section{Figure 5}

Molecular models of beta-sitosterol, naringenin, baicalein, and berberine binding to the predicted target proteins IL-6. (a) IL-6-berberine, (b) IL-6- baicalein, (c) IL-6- naringenin, (d) IL-6- beta-sitosterol

\section{Supplementary Files}

This is a list of supplementary files associated with this preprint. Click to download.

- Supplementary.rar

- Supplementary.rar 